\title{
Sistema de informação para apoio à Sistematização da Assistência de Enfermagem
}

\author{
Information system for supporting the Nursing Care Systematization \\ Sistema de información para apoyo a la Sistematización de la Asistencia de Enfermería
}

\author{
Andreia Malucelli', Kelly Rafaela Otemaier', Marcel Bonnet', Marcia Regina Cubas", Telma Ribeiro Garcia" \\ 'Pontifícia Universidade Católica do Paraná. Programa de Pós-graduação em Informática. Curitiba, PR \\ "Associação Brasileira de Enfermagem. Subcomissão de Sistematização da Assistência de Enfermagem. Brasília, DF
}

Submissão: 26/02/2010

Aprovação: $21 / 07 / 2010$

\section{RESUMO}

A importância, pertinência e necessidade de implantação da Sistematização da Assistência de Enfermagem nos diferentes ambientes da prática profissional é fato ineuestionável. Tendo isso como princípio, objetivou-se elaborar um sistema de informação para apoio à Sistematização da Assistência de Enfermagem, baseado nas etapas do Processo de Enfermagem, nas Necessidades Humanas e utilizando a linguagem de diagnósticos, intervenções e resultados de enfermagem para documentação da prática profissional. Neste artigo, são descritas as etapas metodológicas e os resultados do desenvolvimento do referido sistema de informação - levantamento de reeuisitos, modelagem, mapeamento objeto-relacional, implementação e validação do sistema.

Descritores: Enfermagem; Processos de Enfermagem; Sistemas de Informação.

\section{ABSTRACT}

It is an uneuestionable fact, the importance, relevance and necessity of implementing the Nursing Care Systematization in the different environments of professional practice. Considering it as a principle, emerged the motivation for the development of an information system to support the Nursing Care Systematization, based on Nursing Process steps and Human Needs, using the diagnoses language, nursing interventions and outcomes for professional practice documentation. This paper describes the methodological steps and results of the information system development - requirements elicitation, modeling, object-relational mapping, implementation and system validation. Key words: Nursing; Nursing Process; Information System.

\section{RESUMEN}

La importancia, pertinencia y necesidad de implantación de la Sistematización de la Asistencia de Enfermería en los diferentes ambientes de la práctica profesional es facto incuestionable. Así se objetivó elaborar un sistema de información para apoyo a Sistematización de la Asistencia de Enfermería, basado en las etapas del Proceso de Enfermería, en las Necesidades Humanas e utilizando la lenguaje de diagnósticos, intervenciones y resultados de enfermería para documentación de la práctica profesional. En este artículo, son descriptos las etapas metodológicas y los resultados del desarrollo del referido sistema de información - levantamiento de requisitos, modelaje, mapeamento objeto-intencional, implementación y validación del sistema.

Descriptores: Enfermería; Proceso de Enfermería; Sistema de Información. 


\section{INTRODUÇÃO}

A necessidade de capacitar os profissionais da Enfermagem para uma assistência sistematizada, de Qualidade, tem sido objeto de preocupação, tanto de instituições formadoras Quanto das entidades de classe. A Associação Brasileira de Enfermagem (ABEn), por meio da Subcomissão de Sistematização da Prática de Enfermagem (Portaria No 002/2008, ABEn Nacional, Gestão 2007/2010. Disponível em: http://www.abennacional.org.br/images/conteudo/ PORTARIA_002.pdf), participa do esforço para a implantação da Sistematização da Assistência de Enfermagem (SAE) nas instituições de saúde brasileiras. Parte-se do princípio que a SAE contribui para organizar o cuidado, tornando possível a operacionalização do Processo de Enfermagem e, dessa forma, dando visibilidade à contribuição da Enfermagem no âmbito da atenção à saúde, em QualQuer ambiente onde a prática profissional ocorra, seja em instituições prestadoras de serviços de internação hospitalar, ou em serviços ambulatoriais, escolas, associações comunitárias, fábricas, domicílios, entre outros.

O Processo de Enfermagem requer conhecimento teórico, experiência prática e habilidade intelectual; e indica um conjunto de ações executadas face ao julgamento sobre as necessidades da pessoa, família ou coletividade humana, em determinado momento do processo saúde e doença ${ }^{(1)}$.

Nesse contexto, é preciso ter em conta Que o cuidado profissional de Enfermagem não é um fenômeno natural e, sim, resultante de um empreendimento humano, ou seja, é um instrumental tecnológico desenvolvido ao longo da formação profissional e aperfeiçoado em atividades de educação permanente, Que resultem numa prática reflexiva e crítica dos profissionais da Enfermagem.

O desenvolvimento e o aperfeiçoamento da competência para aplicação, de modo planejado e dinâmico, de um processo assistencial, possibilita identificar, compreender, descrever, explicar e predizer Quais as necessidades da pessoa, família ou coletividade humana, em determinado momento do processo saúde e doença ${ }^{(1)}$.

A SAE e o Processo de Enfermagem, regulamentados na Resolução COFEN n 358/2009, representam uma necessidade colocada cada vez mais frequentemente pelos serviços de saúde. Assim, considerando a importância, pertinência e necessidade de sua implantação nos diferentes ambientes em Que os profissionais da Enfermagem atuam é Que se propôs a elaboração de um sistema de informação para apoio à SAE.

A construção deste sistema de informação, como uma ferramenta para a assistência, gestão e controle social no SUS, faz parte do plano de trabalho da Subcomissão de Sistematização da Prática de Enfermagem, da ABEn Nacional, Gestão 2007-2010. Sua concepção foi atribuída a um grupo de trabalho (Portaria № 00 l/ 2009, ABEn Nacional, Gestão 2007/20 10. Disponível em: http:/ /www.abennacional.org.br/images/conteudo/PORTARIA_00 l.pdf), responsável por definir aspectos fundamentais para a elaboração da modelagem preliminar de um software baseado nas etapas do Processo de Enfermagem, nas Necessidades Humanas e utilizando a linguagem de diagnósticos, intervenções e resultados de enfermagem para documentação da prática profissional.

O desenvolvimento deste sistema de informação resulta de uma parceria entre a ABEn, por intermédio dos membros da Subcomissão de Sistematização da Prática de Enfermagem, e a Pontifícia
Universidade Católica do Paraná (PUCPR), Programa de PósGraduação em Tecnologia em Saúde (PPGTS), envolvendo a participação de docentes e graduandos do curso de Bacharelado em Sistemas de Informação.

Este artigo tem como objetivo descrever as etapas metodológicas e os resultados do desenvolvimento do referido sistema de informação.

\section{ETAPAS METODOLÓGICAS}

Tratou-se do desenvolvimento de um instrumento tecnológico, cuja finalidade seria a de apoiar a SAE, para cujo desenvolvimento foram utilizadas as etapas descritas a seguir.

\section{Levantamento de requisitos}

Foram utilizadas várias técnicas para o levantamento de reQuisitos, dentre as Quais: contatos iniciais, workshop, observação no local e prototipação em papel.

Os contatos iniciais tiveram como finalidade a identificação dos objetivos e restrições do sistema a ser construído. Estes contatos envolveram enfermeiros de unidades básicas de saúde; pesquisadoras e membros da Diretoria Nacional e da Subcomissão de Sistematização da Prática de Enfermagem da ABEn, Gestão 2007/2010, todas com experiência em Processo de Enfermagem e uso de sistemas de classificação dos elementos da prática da Enfermagem. O objetivo foi o de obter informações relevantes a respeito das necessidades dos usuários, de possíveis problemas existentes com o processo de controle e registro do cuidado de Enfermagem à clientela.

A observação visava facilitar o entendimento dos profissionais de sistemas de informação (SI) e garantir Que o sistema atenderia as necessidades reais dos enfermeiros. Assim, os profissionais de SI participaram da rotina de trabalho de enfermeiros, para observar os atendimentos realizados a usuários de uma Unidade de Saúde da cidade de Curitiba-PR; como o trabalho de Enfermagem era realizado e suas dificuldades. A partir desta vivência, foram visualizados Quais requisitos eram importantes; como se utilizava o sistema de informação existente na Unidade de Saúde; que dados eram armazenados e Que relatórios eram emitidos; Que dados e relatórios eram considerados relevantes ou desnecessários; e Que dados, apesar de relevantes, não estavam incluídos. Também foram observadas as dificuldades de usabilidade do sistema implantado, Que contribuíam para certa insatisfação dos profissionais de Enfermagem.

Os workshops envolveram enfermeiras integrantes do grupo de trabalho instituído pela ABEn e outras enfermeiras, de diferentes estados do Brasil, com interesse na SAE e no Processo de Enfermagem, para delimitação de objetivos, funcionalidades e usabilidade do sistema.

Para esboçar a interface do sistema, foram desenhados, previamente ao desenvolvimento, protótipos em papel, de sorte a possibilitar uma discussão da usabilidade do sistema. A prototipação em papel é uma maneira fácil e rápida de criar esboços.

\section{Modelagem do sistema}

O sistema foi modelado utilizando a Unified Modeling Language $(\mathrm{UML})^{(2)}$ com o objetivo de especificar, documentar e visualizar o sistema como um todo. A modelagem foi totalmente baseada nas 
necessidades de informações básicas dos profissionais de Enfermagem Que acompanharam este processo. Foram desenvolvidos os seguintes artefatos: diagrama e especificação de casos de uso, diagrama de classes e diagramas de seeuência.

\section{Mapeamento objeto-relacional}

Como, para a construção do sistema, foi utilizado um Sistema Gerenciador de Banco de Dados (SGBD) relacional, houve necessidade de um mapeamento objeto relacional ${ }^{(3)}$, para facilitar o acesso aos dados e possibilitar várias abordagens em seu tratamento.

\section{Implementação do sistema}

O sistema foi implementado em linguagem de programação Java com arQuitetura cliente-servidor. Isso permite Que suas funcionalidades estejam acessíveis em um navegador de páginas de internet, a partir de um servidor de aplicação na internet e/ou intranet corporativa. Para garantir o tempo de disponibilidade e segurança dos dados, o bem mais precioso gerenciado por este sistema, todo o acesso a eles foi implementado para Que possa operar até mesmo com múltiplos servidores de bancos de dados redundantes e geograficamente distribuídos, usando-se a implementação do EJB3 (Enterprise Java Beans) no servidor de aplicação JBoss e do JPA (Java Persistence API) do Hibernate.

Foi utilizado o SGBD MySQL $5^{(4)}$ por ser robusto e confiável, além de ser gratuito, mas nada impede Que o SGBD seja substituído por Qualquer outro, facilmente, se houver suporte para implementação do Hibernate, o responsável por executar as operações de persistência no banco de dados.

Para obter uma boa usabilidade do sistema e desenvolvimento aliado aos melhores padrões de projeto do mercado, utilizou-se o framework Apache Wicket (http://wicket.apache.org). Para o usuário, o Wicket oferece uma interface rica e poderosa; para o desenvolvedor aumento de produtividade com uma ferramenta de fácil aprendizado e inúmeros recursos - reduzindo o custo sobre o tempo de programação; e do ponto de vista do sistema, o Wicket é capaz de torná-lo robusto, extensível com novas funcionalidades, seguro e compatível com alguns dos melhores recursos de acesso a banco de dados, contudo sem perder em desempenho para outros frameworks disponíveis no mercado.

Também foram criadas folhas de estilos Cascading Style Sheet $(\mathrm{CSS})^{(5)}$ para padronizar layouts e controlar cores, margens, linhas, alturas, larguras e posicionamento de imagens.

\section{Validação do sistema}

Em cada etapa do desenvolvimento, o sistema foi sendo validado pelos profissionais envolvidos no processo de levantamento de requisitos. Um protótipo das telas foi apresentado no $15^{\circ}$ Seminário Nacional de Pesquisa em Enfermagem, realizado no Rio de JaneiroRJ, 2009, tendo havido sugestões de participantes, as Quais foram consideradas em modificações no sistema. O sistema ainda terá Que ser homologado pelos profissionais de Enfermagem envolvidos no processo.

\section{RESULTADOS E DISCUSSÃO}

\section{Levantamento de requisitos}

Optou-se pela construção de uma versão preliminar do sistema com funcionalidades básicas capazes de atender às diversas áreas de atenção e realidades do Brasil para Que, posteriormente, fosse sendo aperfeiçoado e adaptado às realidades e necessidades locais.

O sistema tem como objetivo o apoio à Sistematização da Assistência de Enfermagem no Sistema Único de Saúde; como escopo a coleta de dados, diagnóstico de enfermagem, planejamento da assistência, implementação da assistência e avaliação da assistência; e, como usuários do sistema, Enfermeiros, Auxiliares de Enfermagem, Técnicos de Enfermagem, estudantes de graduação e estudantes do nível médio.

O Quadro 1 apresenta uma lista dos requisitos básicos do sistema, sendo que cada um deles foi descrito e detalhado pelo grupo de enfermeiras envolvidas no levantamento de requisitos.

\section{Modelagem do sistema}

Foram desenvolvidos vários artefatos para permitir o entendimento do domínio de aplicação, documentação e posterior

\begin{tabular}{|c|l|}
\hline Escopo & \multicolumn{1}{|c|}{ Requisitos } \\
\hline \multirow{2}{*}{ Coleta de dados } & $\begin{array}{l}\text { Registrar dados da entrevista } \\
\text { Registrar dados do exame físico } \\
\text { Registrar dados complementares } \\
\text { Possibilitar a importação dos dados do prontuário único }\end{array}$ \\
\hline Diagnóstico de Enfermagem & $\begin{array}{l}\text { Selecionar diagnósticos de enfermagem pré-elaborados por necessidade humana } \\
\text { Possibilitar a formulação de novos diagnósticos, de acordo com o modelo ISO 18.104 }\end{array}$ \\
\hline \multirow{2}{*}{ Planejamento da assistência } & $\begin{array}{l}\text { Possibilitar a seleção de ações pré-elaboradas, por diagnóstico } \\
\text { Possibilitar a formulação de novas ações de enfermagem, de acordo com o modelo ISO } \\
18.104 \\
\text { Possibilitar a formulação de resultados de enfermagem, de acordo com o modelo ISO } \\
18.104\end{array}$ \\
\hline Implementação da assistência & $\begin{array}{l}\text { Registrar as ações realizadas } \\
\text { Registrar informações complementares }\end{array}$ \\
\hline Avaliação da assistência & $\begin{array}{l}\text { Incluir escala para registro da avaliação do alcance de resultados por diagnóstico } \\
\text { Possibilitar o registro de informações complementares }\end{array}$ \\
\hline
\end{tabular}

Quadro 1. Lista de requisitos do sistema. 
manutenção do sistema. Dentre os diagramas, serão apresentados os de casos de uso, de classes e um exemplo dos de sequência.

\section{Diagrama de Casos de Uso}

Os diagramas de casos de uso (Figura 1) são considerados fundamentais na modelagem de sistemas, pois identificam as interações necessárias, descobertas durante o levantamento de requisitos. Nesse diagrama, os usuários, equipamentos, banco de dados, ou mesmo outros sistemas Que possam interagir com o sistema a ser desenvolvido, são representados por atores (stickman); os casos de uso (ações) são representados por elipses; e as interações entre os atores e os casos de uso são representadas por uma linha contínua ${ }^{(6-7)}$.

\section{Diagrama de Classes}

O diagrama de classes (Figura 2) representa as interações estáticas e as classes envolvidas no sistema, permitindo também identificar as hierarquias das classes, representadas por heranças e agregações. Este diagrama permite visualizar os dados Que serão armazenados e manipulados pelo sistema ${ }^{(6,7)}$. Na tentativa de melhorar a sua legibilidade, o diagrama está apresentado parcialmente.

\section{Diagrama de Sequência}

Os diagramas de sequência são elaborados para acrescentar informações aos casos de uso. Esse tipo de diagrama apresenta a dinâmica das operações e interações entre os atores e objetos envolvidos com o sistema ${ }^{(7,8)}$. O diagrama de sequência (Figura 3) é composto por atores, objetos e mensagens, sendo os objetos representados por retângulos sobre uma linha tracejada denominada linha da vida, por representar a vida do objeto na interação do sistema. A mensagem é representada por uma linha com uma seta na ponta, indicando a direção da mensagem entre dois objetos. Este diagrama apresenta as interações para a realização de uma consulta.

\section{Interface do Sistema}

O sistema foi desenvolvido levando em consideração a necessidade de rapidez no acesso às informações, possibilidade de diagnósticos, intervenções e resultados de enfermagem mais precisos e padronizados, relatórios e estatísticas relevantes para obtenção de informações epidemiológicas e facilidade no uso. As principais funcionalidades do sistema são explicadas a seguir, com a respectiva interface.

\section{Pesquisa pessoa}

É uma pesquisa realizada para identificar se uma pessoa já realizou uma consulta anteriormente. A pesquisa é realizada informando um dos seguintes parâmetros: RG, CPF, nome (ou parte do nome) da pessoa, ou nome (ou parte do nome) da mãe da pessoa. Por existir variações de grafia para o mesmo nome, a peseuisa pode ser realizada utilizando a busca fonética conforme apresentado na Figura 4.

\section{Preferência da pesquisa}

Caso a pessoa não esteja cadastrada no sistema, uma tela de preferência é apresentada, Questionando se o nome pesquisado deve ser utilizado para iniciar uma nova investigação (Figura 5).

\section{Identificação}

Refere-se ao registro dos dados pessoais da pessoa (Figura 3). Os campos com uma seta, como nome, sexo, nome da mãe e cor, são de preenchimento obrigatório, todos os demais são opcionais. Esta é a primeira tela do sistema, cujas funcionalidades estão organizadas em abas: Identificação, História, Dados Gerais, Necessidades Humanas, Diagnóstico e Finalização (Figura 6).

\section{História}

Refere-se ao histórico clínico com a intenção de averiguar doenças preexistentes, predisposições, alergias, restrições alimentares, procedimentos realizados e medicações em uso. Para facilitar o preenchimento das informações e evitar telas com muitos dados, a interface foi desenvolvida de maneira a permitir a abertura e fechamento da tela sem sair da funcionalidade História. Em cada fase da História é possível selecionar várias informações como sendo relevantes, objetivando Que estas informações fiquem em destaque nas próximas consultas. O resultado desta seleção pode ser visualizado na funcionalidade Sumário da Pessoa (Figura 7).

\section{Dados Gerais}

São os dados antropométricos e vitais da pessoa (Figura 8). Preenchendo o peso e altura, o IMC (índice de massa corpórea) é calculado automaticamente; preenchendo a pressão sistólica e diastólica, o sistema 


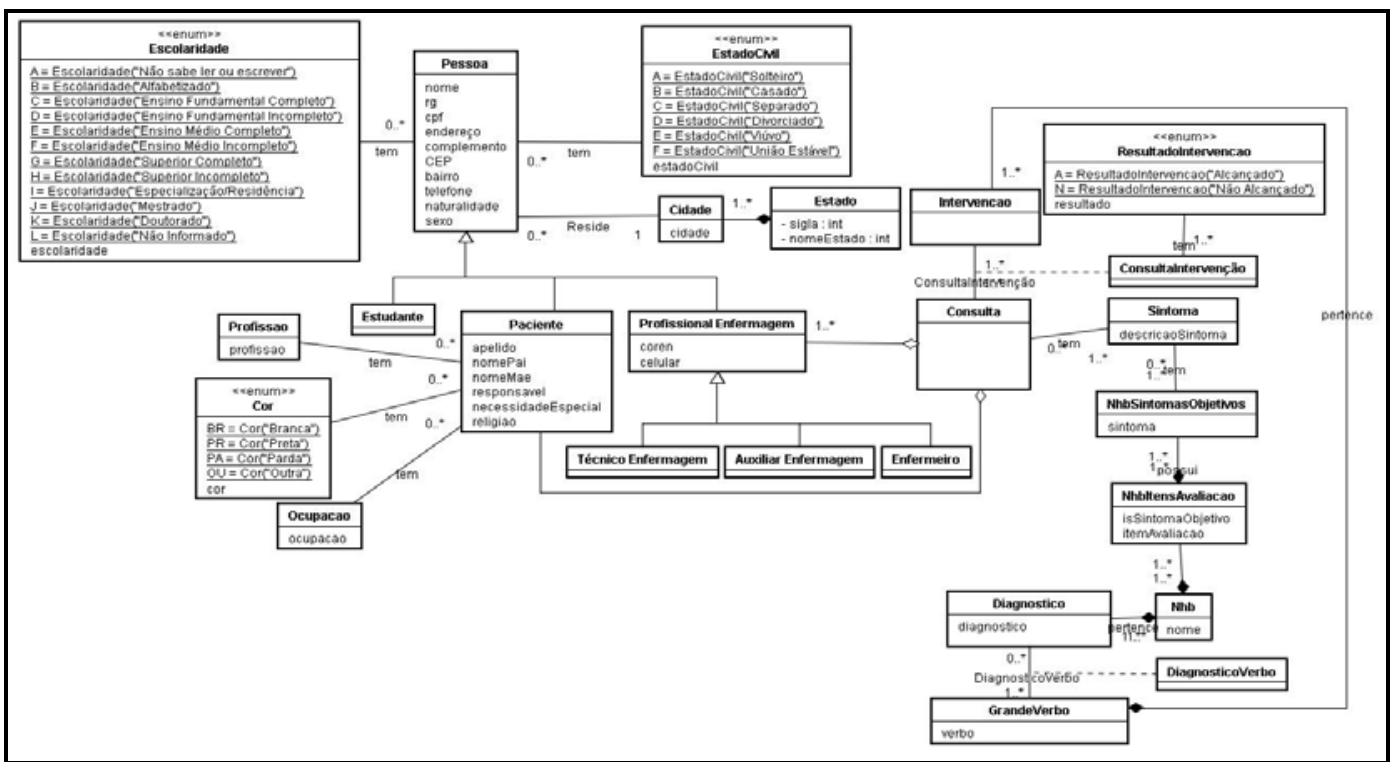

Figura 2. Diagrama de classes conceitual.

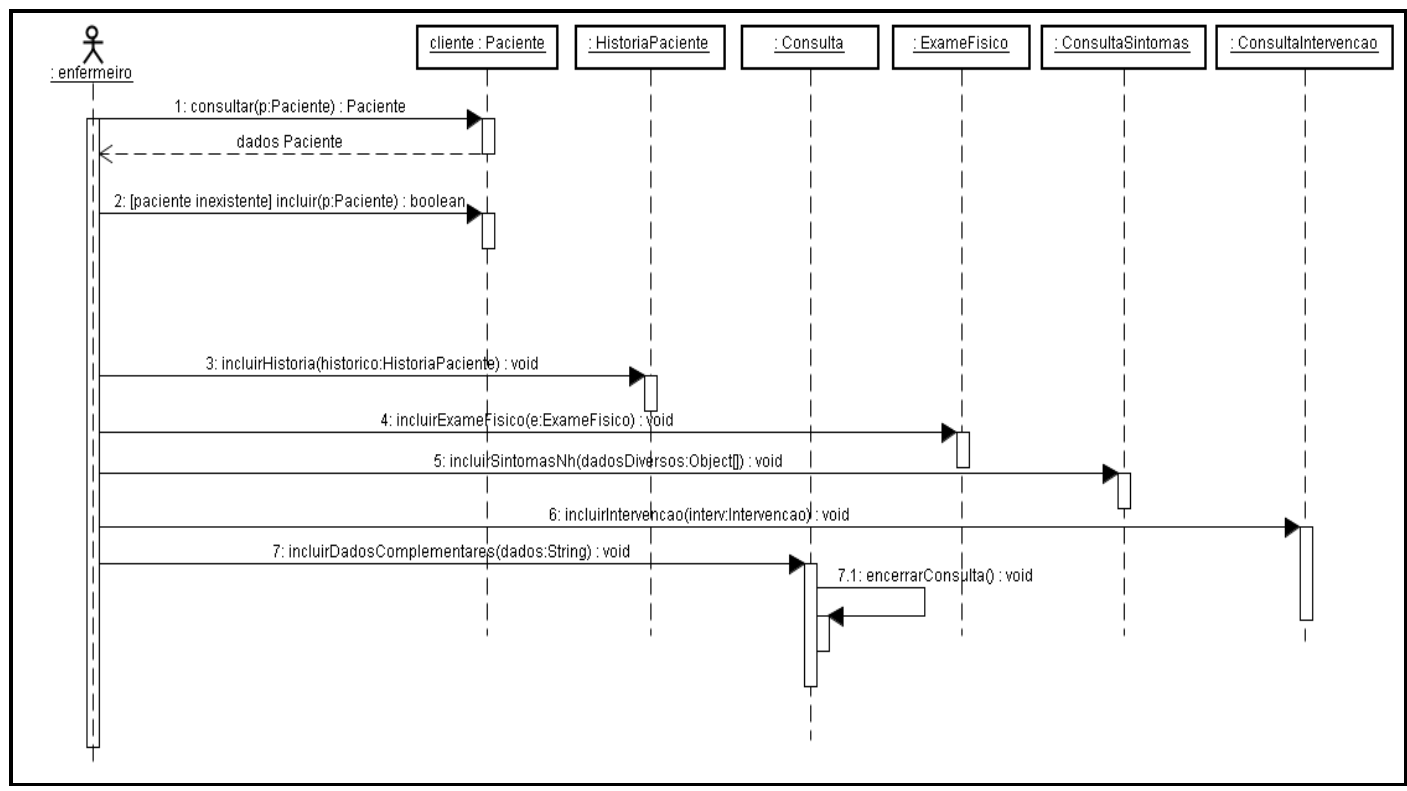

Figura 3. Diagrama de seQuência.

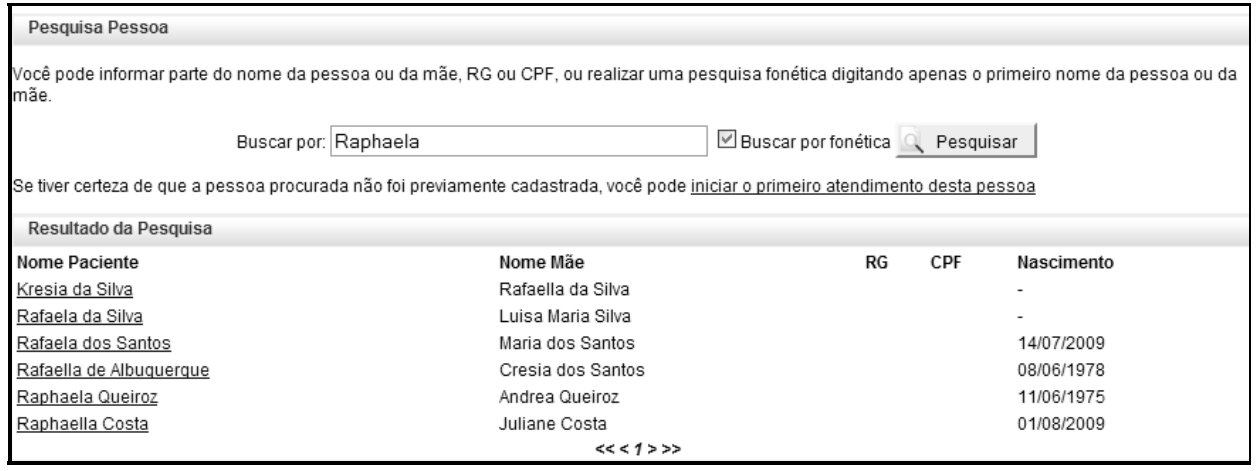

Figura 4. Interface da PesQuisa Pessoa. 


Preferência de Pesquisa do Usuário
$\begin{aligned} & \text { Deseja iniciar a investigação usando o nome da pessoa como foi digitado na pesquisa ou iniciar com um campo } \\ & \text { em branco? O nome digitado foi: "Raphaela" } \\ & \text { Usar o nome como digitado ou Iniciar com o nome em branco } \\ & \text { অ Não me pergunte de novo e use minha opção como padrão para mim }\end{aligned}$

Figura 5. Interface de preferência da pesQuisa.

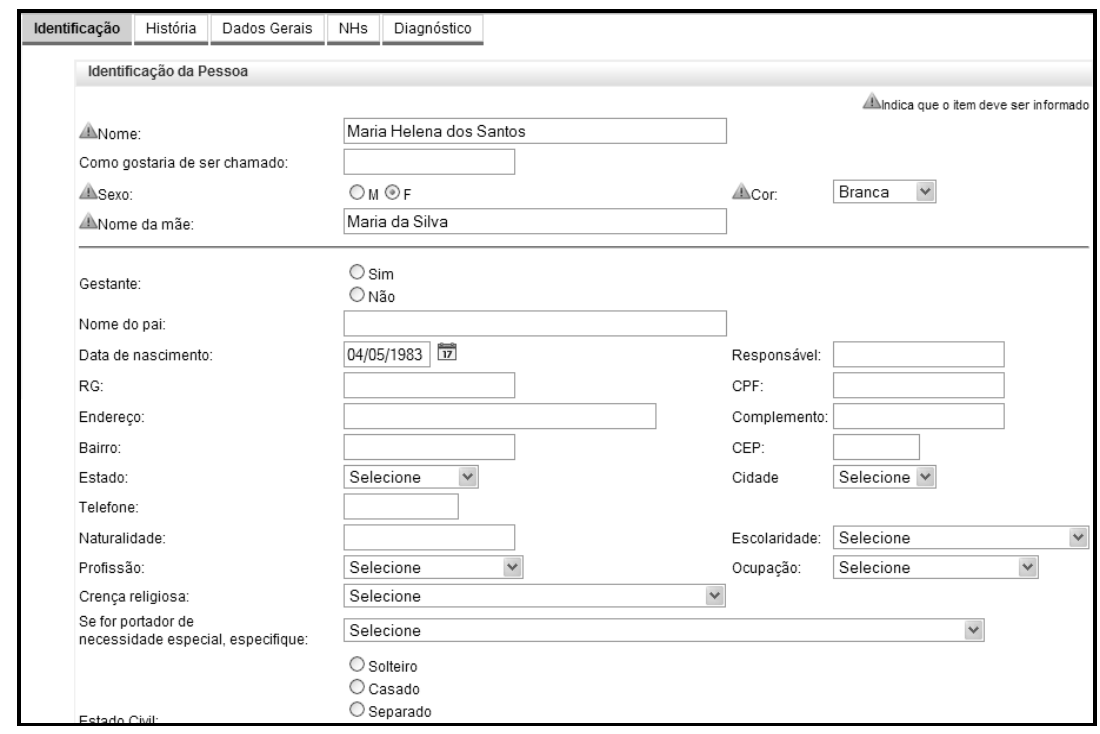

Figura 6. Interface de Identificação da Pessoa.

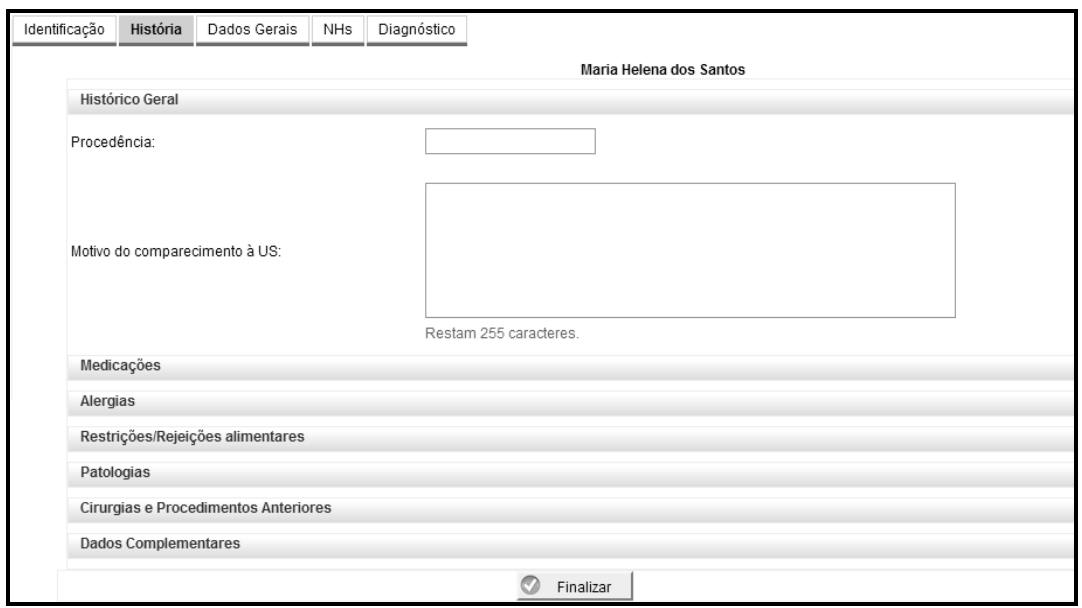

Figura 7. Interface do Histórico da Pessoa.

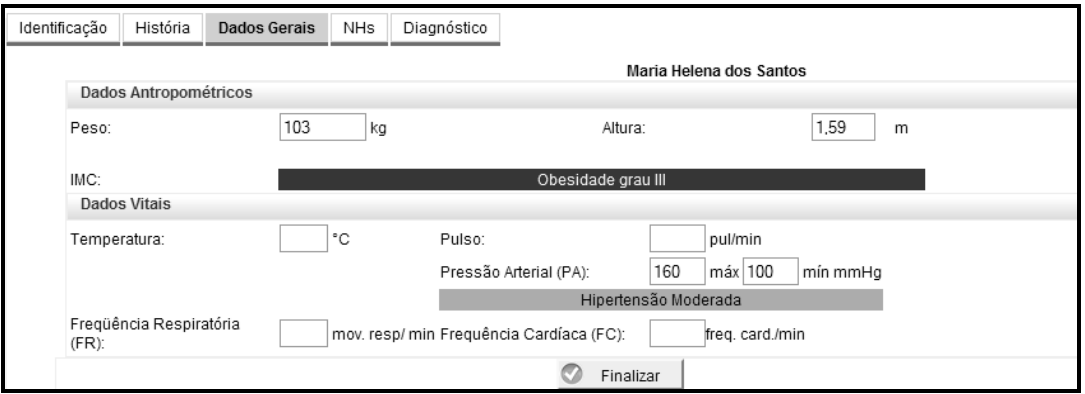

Figura 8. Interface dos dados gerais. 
emite um alerta indicando a avaliação (ótima, normal ou limítrofe) e o estágio da hipertensão (1,2 e 3).

\section{NHs (Necessidades Humanas)}

As necessidades humanas são apresentadas em categorias (Figura 9). Conforme as necessidades humanas são selecionadas, novas abas com o conteúdo dos dados subjetivos e objetivos de cada necessidade humana são adicionadas à página.

\section{Diagnóstico}

O objetivo é auxiliar o enfermeiro a identificar o diagnóstico de enfermagem mais adequado à situação. Esta funcionalidade indica possíveis intervenções para os diagnósticos firmados (Figura 10).

\section{Sumário da Pessoa}

É apresentado um resumo de consultas anteriores e, caso haja uma avaliação de resultado de enfermagem em aberto, um link destacado em vermelho será apresentado na tela (Figura 11).

\section{Resultado da intervenção}

Para cada diagnóstico de enfermagem identificado por Necessidades Humanas deve ser indicado um resultado esperado com a intervenção proposta. Este resultado pode ser avaliado como "Alcançado" ou "Não alcançado" (Figura 12), após o Que se deve clicar no botão "Salvar" para Que fieue registrado. Com isso, o usuário do sistema é redirecionado à página de Resumo da Pessoa, onde poderá ser observado o último resultado de intervenção registrado e um link para iniciar uma nova investigação, caso seja necessário (Figura 13).

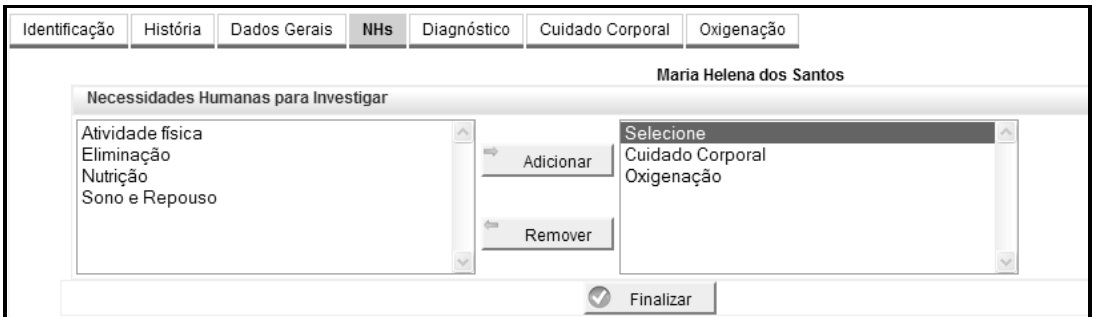

Figura 9. Interface das Necessidades Humanas.

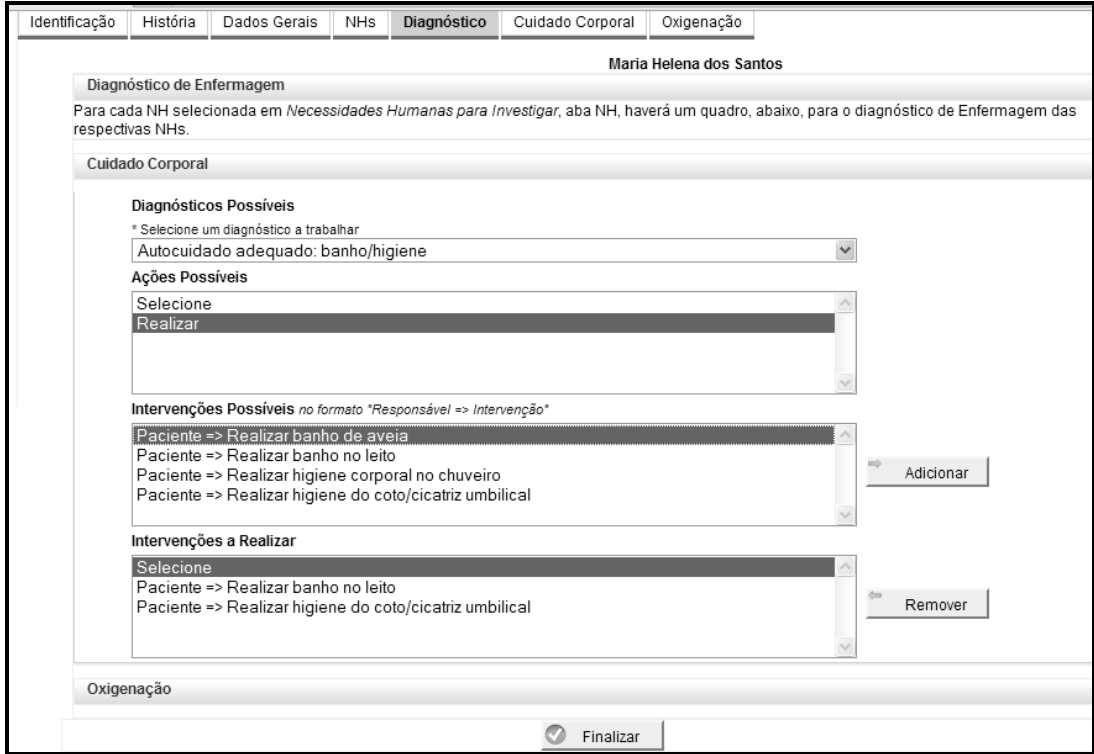

Figura 10. Interface do Diagnóstico.

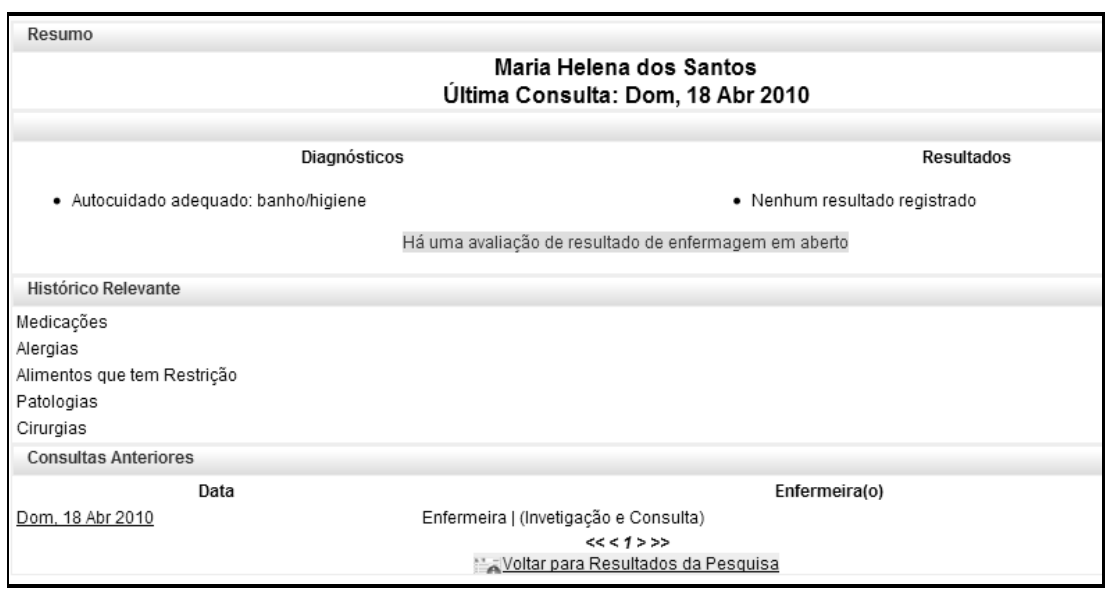

Figura 11. Resumo da pessoa (avaliação de resultado de enfermagem).

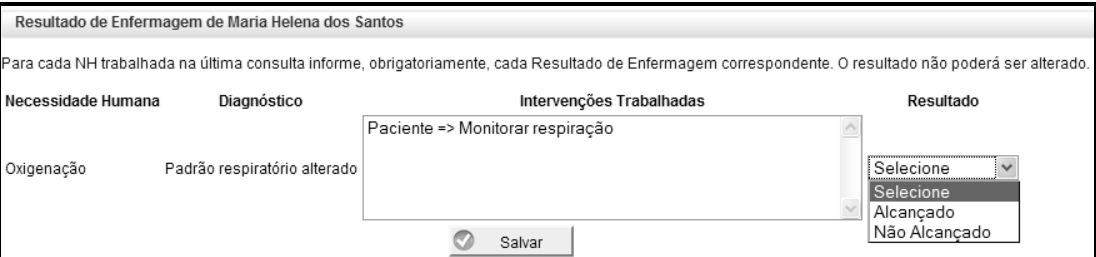

Figura 12. Resultado da intervenção. 


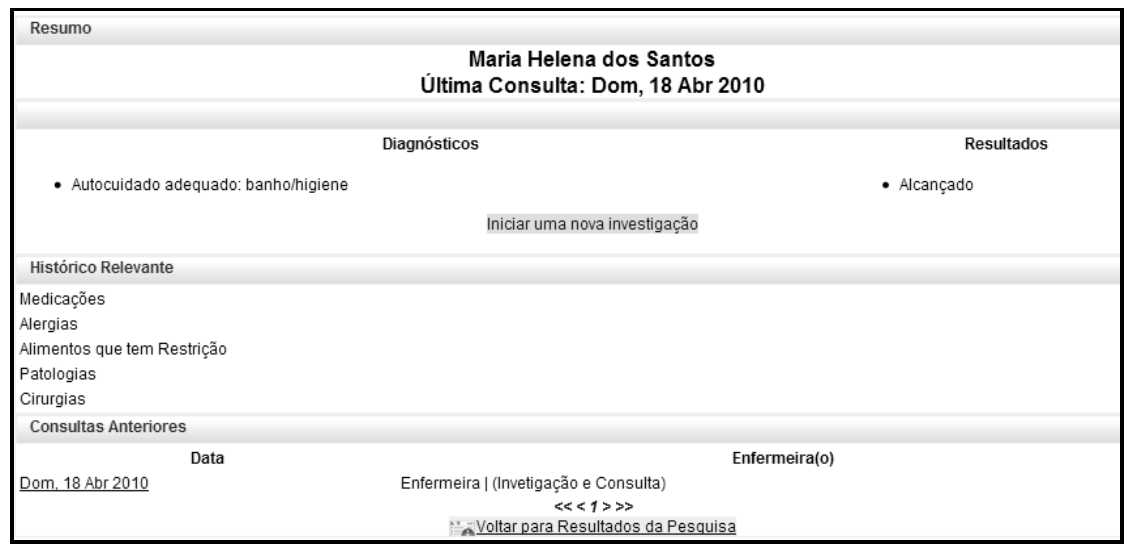

Figura 13. Resumo da pessoa (iniciar nova consulta).

\section{CONSIDERAÇÕES FINAIS}

O sistema de informação apresentado necessita de atualizações e de ser submetido a teste na prática profissional. Neste momento, a principal atualização é a inserção de listagens de diagnósticos/ resultados e intervenções de enfermagem preestabelecidos, bem como a inclusão de alguns itens para coleta de dados.

Ressalta-se a importância da participação efetiva de profissionais da área de Enfermagem na construção de um sistema de informação, em todas as etapas do seu desenvolvimento. Isto torna este sistema mais aderente à prática destes profissionais, assegurando maior usabilidade da ferramenta.

Para os profissionais Que desenvolvem o sistema, a referida participação aumenta a chance de se ter um produto de Qualidade, com conseQuente satisfação do usuário do sistema.
A ABEn, contribuindo para o esforço de implantação da SAE, disponibilizará este sistema em sua página eletrônica para Que os associados e as Escolas e Cursos a ela vinculados utilizem esta ferramenta na formação e em atividade de educação permanente.

\section{Agradecimentos}

Aos membros do grupo técnico de trabalho responsável por definir a modelagem de dados para construção de um sistema de apoio para a Sistematização da Assistência de Enfermagem (Portaria No 00 l/2009, disponível em: http://www.abennacional.org.br/ images/conteudo/PORTARIA_001.pdf)

Às Enfermeiras Lêda Maria Allbuquerque, Carmen Cristina Moura dos Santos, Jussara Gue Martini, Simone Aparecida Peruzzo e Vera Lúcia Mendes Dias.

\section{REFERÊNCIAS}

I. Garcia TR, Nóbrega MML, Carvalho EC. Nursing process: application to the professional practice. Online Braz I Nurs 2004; 3(2). Disponível em: http://www.uff.br/nepae/ objn302garciaetal.htm

2. Object Management Group. Introduction to OMG's - Unified Modeling Language $\mathrm{T}^{\mathrm{TM}}\left(\mathrm{UML}^{\circledR}\right)$. [cited 2009 Aug 25]. Available from: http://www.omg.org/gettingstarted/what_is_uml.htm

3. Mapping Objects to Relational Databases: O/R Mapping In Detail. [cited 2009 Aug 26]. Available from: http:// www.agiledata.org/essays/mappingObjects.html>

4. Why MySQL? [cited 2009 Aug 26]. Available from: http:// www.mysol.com/why-mysol>
5. Web Content Accessibility Guidelines 1.0. [cidet 2009 Aug 27]. Avialable from: http://www.w3.org/TR/I999/WAIWEBCONTENT- I 9990505/\#style-sheet >

6. Sommerville I. Engenharia de software. São Paulo: AddisonWesley; 2003.

7. Von Stein Júnior A, Malucelli A, Bastos LC. Especificação de sistema de informação de microáreas de risco utilizando a abordagem orientada a objetos. Rev Eletr Enferm 2009; I I (4): 866-76. Disponível em: http://www.fen.ufg.br/revista/v I I/n4/ vl In4al2.htm

8. Larman C. Utilizando UML e padrões: uma introdução à análise e ao projeto orientados a objetos e ao desenvolvimento interativo. Porto Alegre: Bookman; 2007. 